\title{
Tenascin-C predicts poor outcomes for patients with colorectal cancer and drives cancer stemness via Hedgehog signaling pathway
}

\author{
Zhaoting Yang ${ }^{1,2}$, Chengye Zhang ${ }^{2}$, Ying Feng ${ }^{1,2}$, Mingji Quan ${ }^{3}$, Yan Cui ${ }^{3^{*}}$ and Yanhua Xuan ${ }^{1,2^{*}}$
}

\begin{abstract}
Background: Tenascin-C (TNC) is an extracellular matrix protein that is widely expressed in the stromal fibroblasts of various cancers. However, the roles of TNC in colorectal cancer (CRC) cells remain unclear.

Methods: The expression of TNC, cancer stem cell-like (CSC) and cell cycle markers, and Hedgehog (HH) signaling pathway genes were assessed in 100 paraffin embedded clinical CRC patient tissues using immunohistochemistry. The interaction between TNC and CSC marker or HH related genes in CRC cells were detected by immunofluorescence. Cell cycle distribution was measured by flow cytometry. Migration and invasion were evaluated by transwell assays. The expressions of TNC, CSC marker, and HH related proteins were analyzed by western blot.
\end{abstract}

Results: TNC expression was markedly upregulated in CRC tissues, and was associated with worse clinical outcomes. TNC overexpression was positively associated with CSC marker LSD1, cell cycle markers CDK4 and p16, and HH signaling pathway related genes SMO and GLI1 in clinical CRC tissue samples. TNC silencing downregulated the expression of the CSC marker LSD1, and the proliferation, migration, and invasion of CRC cells. Interestingly, the GLI1 inhibitor GANT61 strongly inhibited the expression of TNC in CRC cells.

Conclusions: TNC may drive tumor progression and is involved in CSC properties via the HH signaling pathway. TNC has potential value in the evaluation of poor prognosis in CRC.

Keywords: Tenascin-C, Colorectal cancer, Prognosis, Hedgehog signaling pathway

\section{Background}

Colorectal cancer (CRC) is the third leading cause of gastrointestinal cancer-related deaths in the industrialized world [1]. Although the occurrence of CRC has begun to decline in the wealthiest countries, the rate of incidence is still sharply increasing in the developing world [2]. A

\footnotetext{
*Correspondence: cuiyan2005@hotmail.com; xuanyh1@ybu.edu.cn ${ }^{1}$ Department of Pathology, Yanbian University College of Medicine, No. 977, Gongyuan Road, Yanji 133002, China

${ }^{3}$ Department of Oncology, Affiliated Hospital of Yanbian University, No. 1827, Juzi Road, Yanji 133002, China

Full list of author information is available at the end of the article
}

better understanding of the cellular and molecular mechanisms of CRC tumorigenesis would provide insight into the diagnosis and treatment of CRC.

Hedgehog $(\mathrm{HH})$ signaling starts with the secretion of $\mathrm{HH}$ ligand; this is followed by the secretion of Patched (PTC), transmembrane protein Smoothened (SMO), and three GLI zinc finger transcription factors [3]. Of the three GLI proteins, GLI1 is the final and key output of $\mathrm{HH}$ signaling. The HH/GLI1 pathway plays an important role in promoting carcinoma growth, stem cell self-renewal, and metastatic behavior in advanced colon cancers [4]. Human colorectal cancer stem cells 
(CSCs) require active HH/GLI1 signaling for survival and self-renewal [5].

Tenascin-C (TNC) is a large extracellular matrix glycoprotein that is characterized by a six-armed quaternary structure and a modular construction [6]. It is composed of four subunits: a cysteine-rich amino terminal domain, a sequence of epidermal growth factorlike repeats, number of fibronectin type III repeats, and a carboxy-terminal domain homologous to fibrinogen. TNC appears to have supportive roles in tumor growth, metastasis, tumor angiogenesis, and inhibition of immune surveillance [7]. However, overexpression of TNC drives CRC progression by a mechanism that has not yet been elucidated.

In the present study, we demonstrate that TNC expression is significantly correlated with recurrence and poor outcome in CRC. Alteration of TNC expression in CRC cells influences CSC properties, cell proliferation, invasion, and migration. The data highlight a potential $\mathrm{HH}$ signaling pathway for TNC in driving tumor progression, and its potential value in predicting the poor outcomes for patients with CRC.

\section{Materials and methods}

\section{Tissue specimens}

Tissue microarray (human CRC) used for immunohistochemical (IHC) staining was provided by Dr. SeokHyung Kim (Samsung Medical Center, Seoul, South Korea) and the human fetus tissue specimens (used as a positive control of IHC staining) provide by Dr. ZheWu Jin (Wuxi School of Medicine, Jiangnan University, Wuxi, China). All human CRC specimens were collected in Samsung Medical Center (Seoul, South Korea) and the samples use was approved through the Institutional Review Board of Samsung Medical Center (Seoul, South Korea). This research complied with the Helsinki Declaration and was approved by the Institutional Review Board of Samsung Medical Center (No. 201107-122-001). Tissue microarray contains total 100 cases of formalin-fixed and paraffin-embedded CRC tissues samples who underwent curative surgery. No patient received preoperative chemotherapy or radiotherapy. Clinical and pathological reports were reviewed for age, sex, differentiation, clinical stage, pathological tumor (pT) stage, lymph node metastasis, distant metastasis, radiotherapy, chemotherapy and recurrence. The median follow-up period was 112 months (range 2-136 months). The three unstained sagittal sections of human fetus (CRL $53 \mathrm{~mm}$ ) were from the collection of university. The use of specimen for research was approved by the Institutional Review Board of Yanbian University Medical College (No. BS-13-35).

\section{Cell lines}

HT29 and HCT116 cells were maintained in RPMI1640 with high glucose (Life Technologies, Grand Island, NY) supplemented with $10 \%$ heat-inactivated fetal bovine serum (FBS, Life Technologies), $100 \mathrm{mg} /$ $\mathrm{ml}$ penicillin G, and $50 \mathrm{mg} / \mathrm{ml}$ streptomycin (Life Technologies) at $37{ }^{\circ} \mathrm{C}$ in a humidified atmosphere containing $5 \% \mathrm{CO}_{2}$. All cell lines were purchased from ATCC (Manassas, USA). Cells were treated with GANT61 (GAN, ENZO Lifesciences, Rome, Italy) according to the manufacturer's instructions.

\section{Immunohistochemical (IHC) staining procedure}

IHC staining and evaluation of the IHC analysis on CRC tissue microarray samples was performed as previously described [8]. The primary antibody include anti-TNC (1:100, Abcam, UK), anti-SOX2 (1:100, Abcam, UK), anti-CD44 (1:80, Millipore, USA), anti-CD133 (1:100, NOVUS, USA), anti-LSD1 (1:250, Sigma, USA), antiSOX9 (1:100, Abnova, USA), anti-p21 (1:100, Millipore, USA), anti-cyclinD1 (1:100, Millipore, USA), anti-p27 (1:100, Millipore, USA), anti-CDK4 (1:100, Millipore, USA), anti-p16 (1:100, Millipore, USA), anti-SMO (1:250, Santa, USA), and anti-GLI1 (1:100, Abcam, UK).

\section{Immunofluorescence (IF) analysis}

IF procedures were performed according to protocols described previously [9]. The sections were incubated with primary antibodies against TNC (1:50, Abcam, UK), anti-LSD1 (1:50, Sigma, USA) and anti-GLI1 (1:50, Sigma, USA).

\section{Western blotting analysis}

Western blot analysis were carried out according to the standard procedures [8] for anti-TNC (1:1000, Abcam, UK), anti-LSD1 (1:2000, Sigma, USA), anti-SOX2 (1:1000, Abcam, UK), anti-CD44 (1:1000, Millipore, USA), anti-CD133 (1:1000, NOVUS, USA), anti-SOX9 (1:1000, Abnova, USA), anti-GLI1 (1:1000, Abcam, UK), and anti- $\beta$-actin (1:500, Bioss, China).

\section{Cell transfection}

Three different TNC small interfering RNA (siRNA, s7068, s7069 and s7070) targeting HT29 and HCT116 was designed and synthesized by Ambion (Life Technologies, Carlsbad, USA). The sequence of each TNC siRNA was listed in Additional file 2: Table S1. For the drug treatments, we dissolved TNC siRNAs in Nuclease-free Water and diluted the solutions to the $50 \mu \mathrm{M}$. $2 \times 10^{5}$ cells were plated into 6 -well plates with $2 \mathrm{ml}$ of culture medium. After $24 \mathrm{~h}$, the cells were transfected with 100 pmol TNC siRNA using $5 \mu \mathrm{l} /$ well 
Lipofectamine 2000 (Invitrogen, Life Technologies, Grand Island, NY) according to the manufacturer's instructions. After $48 \mathrm{~h}$, total protein was collected to determine the TNC knockdown efficiency by western blotting.

\section{Migration and invasion, and cell cycle analysis}

All migration and invasion, and cell cycle analysis procedures were performed according to protocols described previously [9] for transfecting HT29 and HCT116 with control siRNA or TNC siRNA.

\section{Statistical analysis}

Correlations were examined using Pearson's Chi square test or Fisher's exact test as appropriate. Overall survival (OS) and disease free survival (DFS) were determined using the Kaplan-Meier method and were compared using the log-rank test. Survival was measured from the date of surgery. The Cox proportional hazards model was used for multivariate analysis. Clinicopathologic factors, which were statistically significant in univariate analysis, were included as covariables in multivariate analysis. Hazard ratio (HR) and 95\% confidence intervals (CI) were assessed for each factor. The data are expressed as the mean \pm standard deviation (SD). Comparisons between groups were analyzed using Student's t-test or ANOVA. All tests were two sided, and $P<0.05$ was considered significant. The statistical analysis was performed using GraphPad Prism 7.04 (GraphPad Software, Inc., La Jolla, CA, USA) and SPSS 19.0 statistical software (SPSS Inc, Chicago, IL, USA).

\section{Results}

\section{TNC expression is correlated with poor clinical outcomes} in CRC

TNC was found to be primarily expressed in the colon of the human fetus (Fig. 1a) and CRC tissues (Fig. 1d). Positive signals of TNC expression were mainly localized in the nuclei and cytoplasm of epithelial cells, as well as the cytoplasm of fibroblasts of colorectal tissue (Fig. 1a-d). TNC in cancer cells was significantly associated with tumor recurrence $(P=0.034)$. There were no significant associations of TNC expression with other clinicopathological features (Additional file 2: Table S2).

Kaplan-Meier survival analysis revealed that CRC patients with high TNC expression had significantly shorter 5-year OS ( $P=0.007$, Fig. 1e) and DFS $(P=0.004$, Fig. 1f). Univariate Cox regression analysis revealed factors that were independently associated with poor prognosis for both OS and DFS. These factors were $\mathrm{pT}$ stage $(P<0.001$ for OS; $P<0.001$ for DFS), lymph node metastasis $(P=0.001$ for OS; $P<0.001$ for DFS), distant metastasis $(P<0.001$ for OS; $P<0.001$ for DFS $)$, and TNC expression $(P=0.008$ for OS; $P=0.011$ for DFS) (Table 1$)$. Multivariate Cox regression analysis revealed the independent association with poor prognosis of OS and DFS for distant metastasis $(P=0.004$ for OS; $P=0.008$ for DFS) and TNC expression ( $P=0.001$ for OS; $P=0.002$ for DFS) (Table 2).

\section{TNC regulates CSC marker LSD1 in CRC}

To determine whether TNC regulates the stemness for CRC cells, we studied the association between TNC and the hallmark CSC genes CD133, CD44, LSD1, SOX2, and SOX9. TNC was positively correlated with LSD1 protein expression $(P=0.007$, Table 3$)$ and was co-expressed in the same sections of CRC tissues (Fig. 2a, b). Furthermore, the TNC positive cell population markedly overlapped with the LSD1 positive cell population in HT29 (Fig. 2c) and HCT116 cells (Fig. 2d). CD133, CD44, SOX2, and SOX9 were co-upregulated with TNC in HCT116 cells compared to HT29 cells (Additional file 1: Fig. S1).

Based on these observations, we examined the loss of function to further elucidate the correlation between TNC and cancer stemness. Cells were knocked down for TNC expression using siRNA, including s7068, s7069 and s7070 in HT29 (Fig. 3a) and HCT116 cells (Fig. 3b). Of these, s7068 was the most markedly affected and was selected to establish TNC siRNA cells; this led to low levels of TNC. TNC knockdown was associated with dramatically decreased LSD1 expression compared to that in control group in HT29 cells (Fig. 3c) and HCT116 cells (Fig. 3d).

\section{TNC alters proliferation, migration, and invasion of CRC cells}

To clarify the role of TNC in CRC cell proliferation, we analyzed the relationship between TNC expression and cell cycle markers (p21, cyclinD1, p27, CDK4, and p16) in CRC tissues samples using IHC analysis (Fig. 4a). TNC also appeared to promote CRC cell proliferation and progression as it was positively correlated with the expression of CDK4 $(P=0.025)$ and p16 $(P=0.013)$ (Additional file 2: Table S3). Moreover, compared with the control group, a decrease in S-phase subpopulations and an increase in $G_{0} / G_{1}$-phase subpopulations were observed in TNC siRNA-silenced HT29 cells (Fig. 4b) and HCT116 cells (Fig. 4c). Cell invasion and migration were significantly decreased in the TNC siRNA group compared with the control groups in both HT29 (Fig. 5a) and HCT116 cells (Fig. 5b).

$\mathrm{HH}$ signaling pathway strongly influences TNC expression TNC expression was significantly associated with SMO $(P=0.011)$ and GLI1 $(P<0.001)$ (Table 4, Fig. 6a). There 


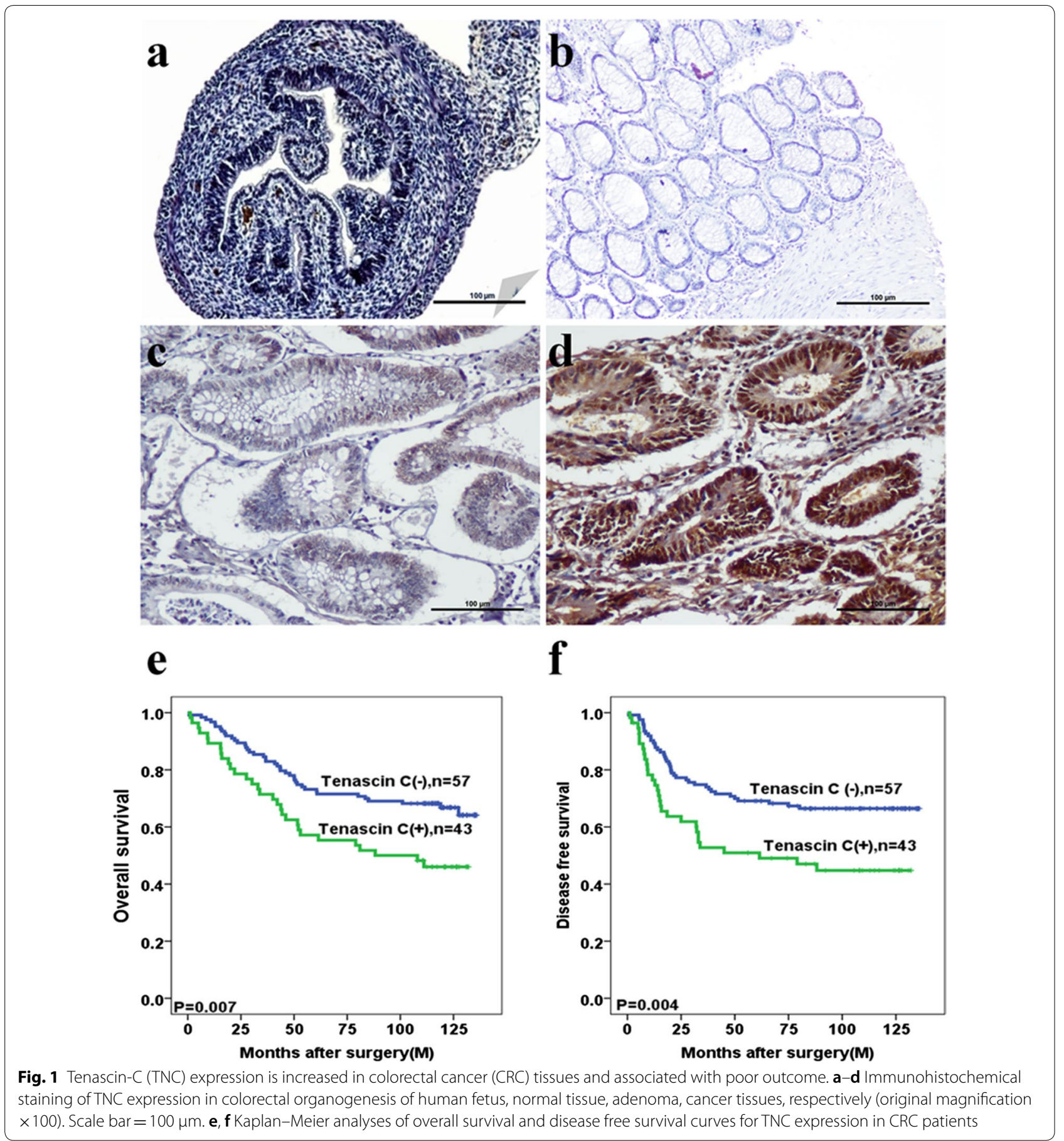

was a considerable overlap between the TNC positive and GLI1 positive cell populations for both HT29 (Fig. 6b) and HCT116 (Fig. 6c) cells by IF staining. In addition, to determine how $\mathrm{HH}$ signaling controls TNC, CRC cells were treated with the GLI1 inhibitor GAN $(20 \mu \mathrm{M})$. The results showed that GAN dramatically downregulated the expression of TNC (Fig. 6d, e).

\section{Discussion}

TNC overexpression predicted poor outcomes in CRC patients and was significantly associated with CSC markers, cell proliferation, and the $\mathrm{HH}$ signaling pathway. TNC silence inhibited the expression of the CSC marker LSD1, and the proliferation and invasion of CRC cells. The association with the $\mathrm{HH}$ signaling pathway may help 
Table 1 Univariate analyses for prognostic variables of overall survival and disease free survival in colorectal cancer patients using Cox proportional-hazards regression

\begin{tabular}{|c|c|c|c|c|c|c|}
\hline \multirow[t]{2}{*}{ Characteristic } & \multicolumn{3}{|c|}{ Overall survival } & \multicolumn{3}{|c|}{ Disease-free survival } \\
\hline & HR & $95 \% \mathrm{Cl}$ & $P$ & $\overline{\mathrm{HR}}$ & $95 \% \mathrm{Cl}$ & $P$ \\
\hline Age (years) & & & 0.388 & & & 0.889 \\
\hline$\leqq 60$ & 1.0 & & & 1.0 & & \\
\hline$>60$ & 1.221 & $0.776-1.921$ & & 0.967 & $0.607-1.542$ & \\
\hline Tstage & & & $<0.001$ & & & $<0.001$ \\
\hline 1 & 1.0 & & & 1.0 & & \\
\hline 2 & 2.281 & $0.796-6.537$ & & 1.404 & $0.529-3.726$ & \\
\hline 3 & 3.129 & $1.078-9.084$ & & 2.654 & $1.008-6.988$ & \\
\hline 4 & 7.692 & $2.653-22.301$ & & 5.250 & $1.980-13.925$ & \\
\hline Lymph node metastasis & & & 0.001 & & & $<0.001$ \\
\hline Negative & 1.0 & & & 1.0 & & \\
\hline Positive & 2.255 & $1.415-3.593$ & & 2.791 & $1.704-4.570$ & \\
\hline Distant metastasis & & & $<0.001$ & & & $<0.001$ \\
\hline Negative & 1.0 & & & 1.0 & & \\
\hline Positive & 5.266 & $2.966-9.351$ & & 5.111 & $2.806-9.307$ & \\
\hline TNC & & & 0.008 & & & 0.011 \\
\hline Negative & 1.0 & & & 1.0 & & \\
\hline Positive & 1.894 & $1.182-3.035$ & & 1.893 & $1.158-3.094$ & \\
\hline
\end{tabular}

Table 2 Multivariate analyses for prognostic variables of overall survival and disease free survival in colorectal cancer patients using Cox proportional-hazards regression

\begin{tabular}{|c|c|c|c|c|c|c|}
\hline \multirow[t]{2}{*}{ Characteristic } & \multicolumn{3}{|c|}{ Overall survival } & \multicolumn{3}{|c|}{ Disease-free survival } \\
\hline & HR & $95 \% \mathrm{Cl}$ & $P$-value & HR & $95 \% \mathrm{Cl}$ & $P$-value \\
\hline Age (years) & & & 0.107 & & & 0.606 \\
\hline$\leqq 60$ & 1.0 & & & 1.0 & & \\
\hline$>60$ & 1.506 & $0.916-2.477$ & & 1.141 & $0.691-1.883$ & \\
\hline Tstage & & & 0.131 & & & 0.641 \\
\hline 1 & 1.0 & & & 1.0 & & \\
\hline 2 & 2.461 & $0.836-7.242$ & & 1.530 & $0.553-4.233$ & \\
\hline 3 & 1.964 & $0.415-9.286$ & & 1.204 & $0.249-5.811$ & \\
\hline 4 & 3.839 & $0.682-21.604$ & & 1.796 & $0.324-9.942$ & \\
\hline Lymph node metastasis & & & 0.419 & & & 0.187 \\
\hline Negative & 1.0 & & & 1.0 & & \\
\hline Positive & 1.613 & $0.506-5.142$ & & 2.308 & $0.667-7.987$ & \\
\hline Distant metastasis & & & 0.004 & & & 0.008 \\
\hline Negative & 1.0 & & & 1.0 & & \\
\hline Positive & 3.307 & $1.466-7.462$ & & 3.230 & $1.365-7.642$ & \\
\hline TNC & & & 0.001 & & & 0.002 \\
\hline Negative & 1.0 & & & 1.0 & & \\
\hline Positive & 2.192 & $1.356-3.545$ & & 2.234 & $1.353-3.688$ & \\
\hline
\end{tabular}


Table 3 The association between protein expression of TNC and cancer stem cell makers in colorectal cancer

\begin{tabular}{|c|c|c|c|c|c|c|}
\hline Variable & $n$ & TNC (-)n (\%) & TNC (+)n (\%) & $x^{2}$ & $R$ & $P$ \\
\hline CD133 & & & & 0.019 & -0.014 & 0.891 \\
\hline Negative & 18 & $10(55.6)$ & $8(44.4)$ & & & \\
\hline Positive & 82 & $47(57.3)$ & $35(42.7)$ & & & \\
\hline CD44 & & & & 0.532 & 0.073 & 0.466 \\
\hline Negative & 69 & $41(59.4)$ & $28(40.6)$ & & & \\
\hline Positive & 31 & $16(51.6)$ & 15 (48.4) & & & \\
\hline LSD1 & & & & 4.910 & 0.222 & 0.027 \\
\hline Negative & 25 & $19(76.0)$ & $6(24.0)$ & & & \\
\hline Positive & 75 & $38(50.7)$ & $37(49.3)$ & & & \\
\hline sox2 & & & & 0.041 & 0.020 & 0.840 \\
\hline Negative & 10 & $6(60.0)$ & $4(40.0)$ & & & \\
\hline Positive & 90 & $51(55.7)$ & 39 (43.3) & & & \\
\hline sox9 & & & & 0.639 & 0.080 & 0.424 \\
\hline Negative & 7 & $5(71.4)$ & $2(28.6)$ & & & \\
\hline Positive & 93 & $52(55.9)$ & $41(44.1)$ & & & \\
\hline
\end{tabular}

a

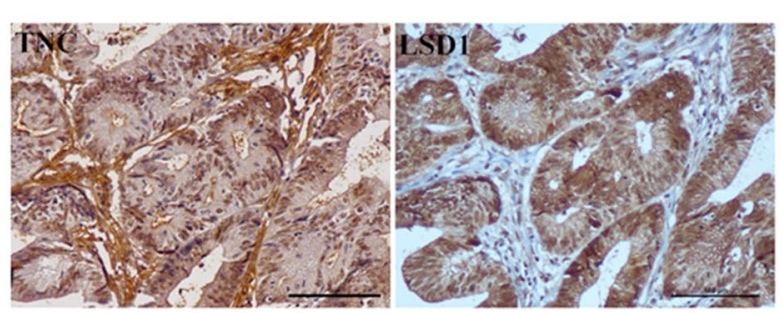

b


Fig. 2 Tenascin-C (TNC) promotes the stemness of colorectal cancer (CRC) cells. a Immunohistochemical staining of CRC with TNC and LSD1 in serial section of CRC tissues (original magnification $\times 200$ ). Scale bar $=100 \mu \mathrm{m}$. b Immunofluorescence (IF) staining for TNC and LSD1 in CRC tissues. c, d IF staining for TNC and LSD1 in the HT29 and HCT116 cells. Blue for DAP1; green for LSD1; red for TNC; double labeling for Merge 

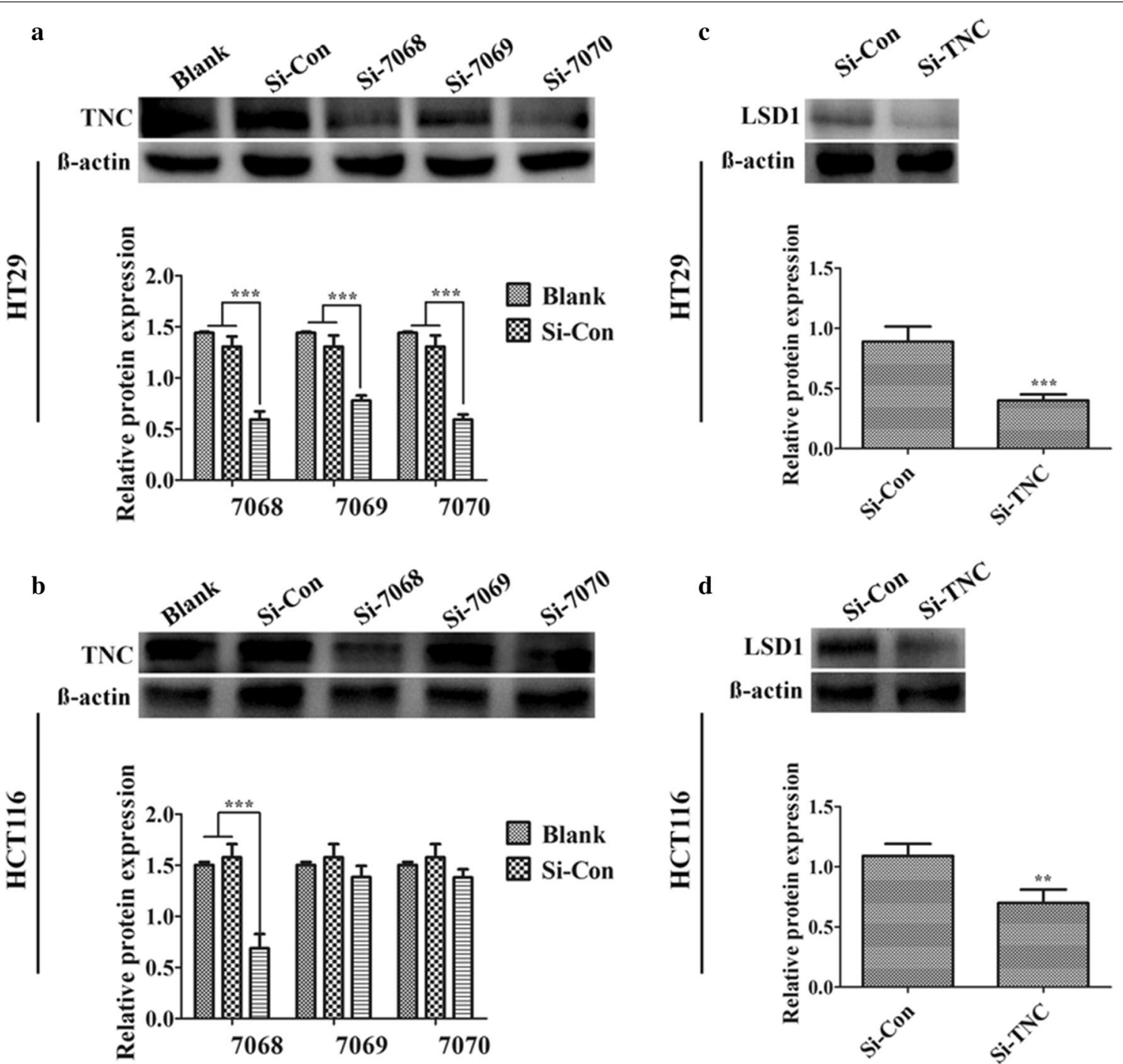

Fig. 3 Tenascin-C (TNC) knockdown exhibited dramatically decreased LSD1 expression. a, b Protein expression of TNC in the HT29 and HCT116 cells after transfecting with nuclease-free water (blank control), control siRNA and TNC siRNA (s7068, s7069 and s7070) for 48 h was confirmed by western blotting analysis. c, d Protein expression of LSD1 in the HT29 and HCT116 cells after transfecting with TNC siRNA compared with control siRNA. Western blotting data were normalized to those for $\beta$-actin and expressed as fold changes relative to levels in the control group. ${ }^{* *} P<0.01$, ***P $P 0.001$ versus control

clarify the correlation between TNC and the progression of CRC.

TNC is transiently expressed in the colon of the human fetus and is re-expressed in pathological conditions like CRC. Increased TNC expression in cancer cells has also been also correlated with the recurrence of human CRC. Parekh et al. studied a small cohort of ten patients with lung cancer and found elevated TNC mRNA and protein expression in patients with early recurrence of the disease [10]. The prior and present observations indicate that
TNC may be involved in CRC tumorigenesis and recurrence. The present finding that elevated TNC expression was significantly correlated with poor outcomes in CRC is consistent with the results of previous studies in patients with esophageal squamous cell carcinoma [11].

CSCs use TNC to block T-cell activation during priming/restimulation. Prostate CSC overexpresses TNC, and TNC silencing abrogates CSC-mediated immunosuppression [12]. LSD1 and its inhibitor have been reported to target CSC markers in several cancers [13-15]. In the 


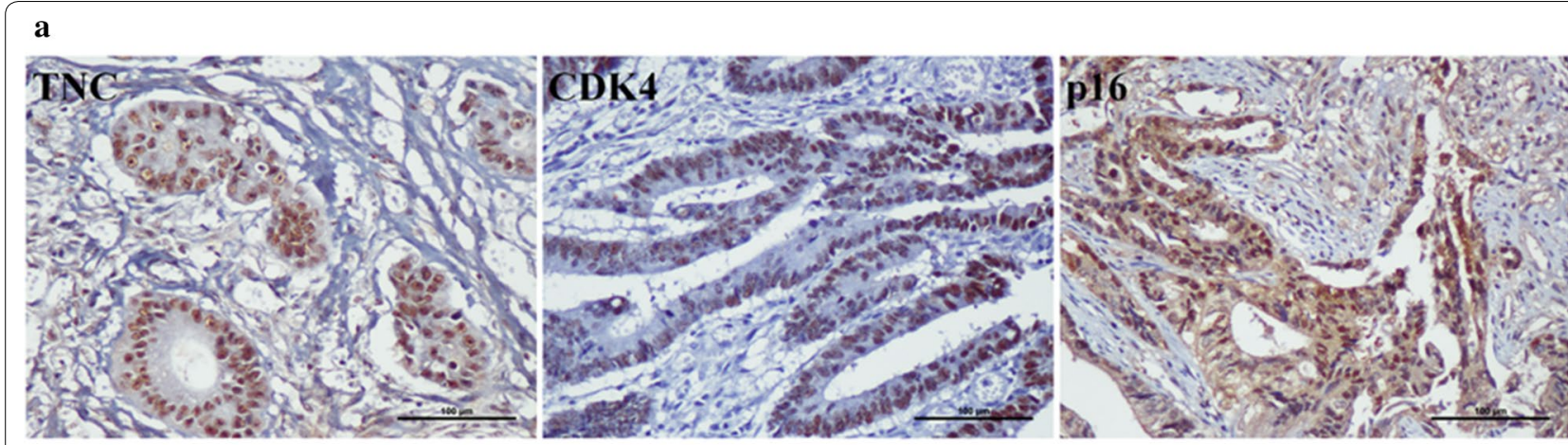

b

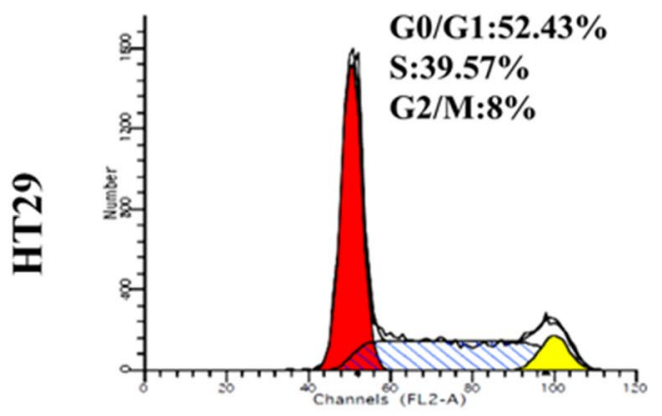

si-Con

c

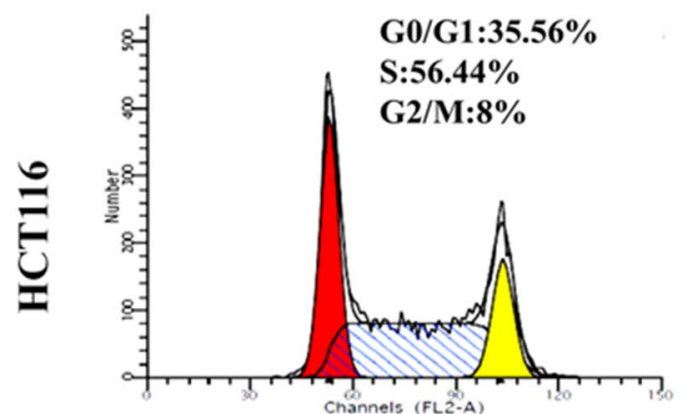

si-TNC



si-TNC



Fig. 4 The over-expression of Tenascin-C (TNC) induced cancer cell proliferation in colorectal cancer (CRC). a Immunohistochemical staining of TNC, CDK4 and p16 in CRC tissues (original magnification $\times 200$ ). Scale bar $=100 \mu \mathrm{m}$. b, c HT29 and HCT116 cells knockdown with TNC siRNA in the cell phases G0/G1, S and G2/M compared with control siRNA group using flow cytometry

present study, we found that the TNC positive cell population overlapped with the LSD1 positive cell population in CRC cells and in serial sections of CRC tissues. Furthermore, we found that TNC knockdown significantly downregulated LSD1 expression. Other makers were coupregulated with TNC in HCT116 cells. Many therapies that have not produced a statistically significant benefit in previous trials may have used inadequate samples, and dismissal of these therapies may have been premature.
We suggest the association of TNC overexpression with poor prognosis in CRC may be present because TNC positively regulates cell stemness. Consistent with the present findings, a recent report suggested that TNC may be a potential prognostic marker for glioblastoma multiforme as well as a potential marker for glioma CSCs [16].

Cai et al. demonstrated that TNC was essential to trigger migration and invasion in pancreatic cancer both in vitro and in vivo [17]. Similarly, the CDK4 and 

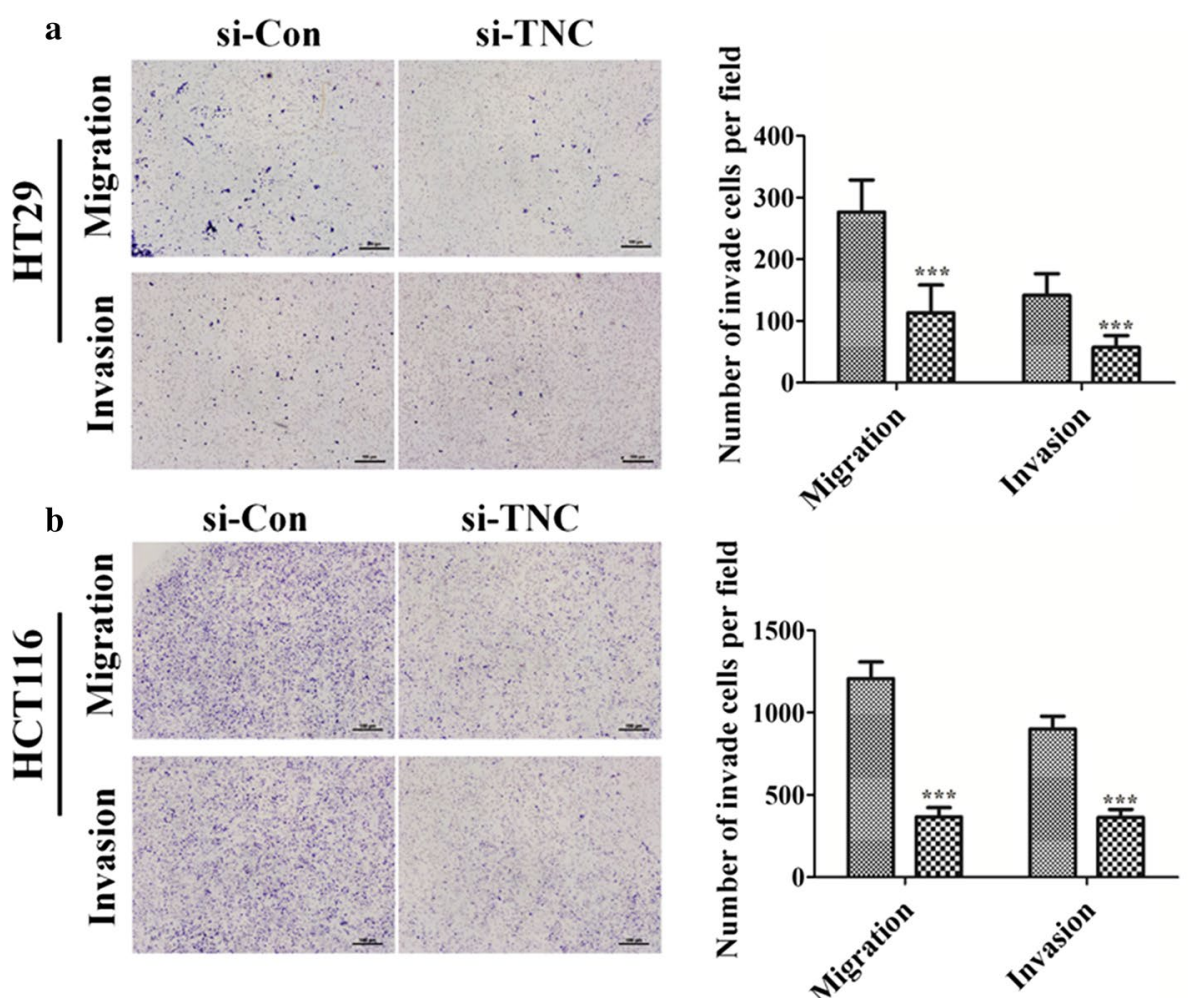

Fig. 5 Tenascin-C (TNC) high-expression up-regulated cancer cell the ability of migration/invasion in colorectal cancer. a, b HT29 and HCT116 cells migration/invasion was investigated by transwell assay after transfecting $48 \mathrm{~h}$ with control siRNA and TNC siRNA. *** $P<0.001$ versus control

p16 cell cycle markers were significantly related to TNC in CRC cells. Furthermore, knockdown of TNC expression in CRC cells significantly suppressed cell proliferation, and impaired the migration and invasion of CRC cells. These findings suggest that the expression of TNC may lead to unrestrained CRC cell proliferation and could influence cell migration and invasion.

Santini et al. reported that blockade of the HH pathway in melanoma stem cells via the chemical and genetic inhibition of SMO and GLI1 effectively reduced self-renewal and tumor-initiation ability of the cells [18]. The expression of TNC was strongly associated with $\mathrm{HH}$ pathway in clinical CRC tissues. Inhibition of the $\mathrm{HH}$ pathway could decrease TNC expression in CRC cells. Thus, $\mathrm{HH}$ signaling may provide an upstream signal for TNC, and might be involved in the targeted activation of TNC to promote colorectal CSC properties.

\section{Conclusions}

Expression of TNC in cancer cells might be a potential prognostic biomarker in patients with CRC. The present results highlight a potential role for TNC in CSC features and provide novel mechanistic insights into the roles of $\mathrm{HH}$ and TNC in driving CRC progression. Our findings suggest that TNC could be a critical target gene for the treatment of CRC.

Table 4 The association between protein expression of TNC and Hedgehog signaling pathway in colorectal cancer

\begin{tabular}{|c|c|c|c|c|c|c|}
\hline Variable & $n$ & TNC (-)n (\%) & TNC (+)n (\%) & $x^{2}$ & $R$ & $P$ \\
\hline $\mathrm{SMO}$ & & & & 5.803 & 0.241 & 0.016 \\
\hline Negative & 44 & $31(70.5)$ & $13(29.5)$ & & & \\
\hline Positive & 56 & $26(46.4)$ & $30(53.5)$ & & & \\
\hline GLI1 & & & & 12.562 & 0.354 & $<0.001$ \\
\hline Negative & 41 & $32(78.1)$ & $9(21.9)$ & & & \\
\hline Positive & 59 & $25(42.3)$ & 34 (57.6) & & & \\
\hline
\end{tabular}









\section{Supplementary information}

Supplementary information accompanies this paper at https://doi. org/10.1186/s12935-020-01188-w.

Additional file 1: Fig. S1. Cancer stem-like cell makers were co-upregulated with TNC in HCT116 cells compared to HT29 cells. Western blotting data were normalized to those for $\beta$-actin.

Additional file 2: Table S1. Sequences of TNC siRNA targeting colorectal cancer cells. Table S2. Comparison of clinicopathologic characteristics according to TNC expression in colorectal cancer. Table S3. The association between protein TNC and cell cycle markers in colorectal cancer.

\section{Abbreviations}

TNC: Tenascin-C; CRC: Colorectal cancer; CSC: Cancer stem cell-like; HH: Hedgehog; SMO: Smoothened; pT stage: Pathological tumor stage; IHC: Immunohistochemical; IF: Immunofluorescence; OS: Overall survival; DFS: Disease free survival; HR: Hazard ratio; Cl: Confidence intervals; SD: Standard deviation; FBS: Fetal bovine serum; GAN: GANT61.

\section{Acknowledgements}

Special thanks to Dr. Zhe-Wu Jin (Department of Anatomy, Wuxi School of Medicine, Jiangnan University, Wuxi, China) provided the human fetus tissue specimens and Dr. Seok-Hyung Kim (Department of Pathology, Samsung Medical Center, Sungkyunkwan University School of Medicine, Seoul, South Korea) provided the colorectal cancer tissue specimens.

\section{Authors' contributions}

$Y X$ and $Z Y$ designed the study. $Y C$ and $Y X$ provided the funds. $Z Y, Y F$ and $M Q$ performed all experiments. YX and CZ performed the statistical analysis. ZY wrote the manuscript. YX is the guarantor of this work. All authors read and approved the final manuscript.

\section{Funding}

This study was supported by grants from National Natural Science Funds of China (81760531).

\section{Availability of data and materials}

The data of the study are available from the corresponding author on reasonable request.

\section{Ethics approval and consent to participate}

This research complied with the Helsinki Declaration and was approved by the Institutional Review Board of Samsung Medical Center (No. 2011-07-122-001). All CRC patients provided written informed consent according to institutional guidelines. Patients were informed that the resected specimens were stored by the hospital and potentially used for scientific research, and that their privacy would be maintained. Follow-up survival data were collected retrospectively through medical record analyses. The human fetus tissue specimens were procured from voluntary pregnancy terminations after surgery and proper consent from donors with the approval of the Institutional Review Board of Yanbian University Medical College (No. BS-13-35).

\section{Consent for publication}

Not applicable.

\section{Competing interests}

No potential conflicts of interests were disclosed.

\section{Author details}

1 Department of Pathology, Yanbian University College of Medicine, No. 977, Gongyuan Road, Yanji 133002, China. ${ }^{2}$ Institute for Regenerative Medicine, Yanbian University College of Medicine, No. 977, Gongyuan Road,
Yanji 133002, China. ${ }^{3}$ Department of Oncology, Affiliated Hospital of Yanbian University, No. 1827, Juzi Road, Yanji 133002, China.

Received: 1 April 2019 Accepted: 27 March 2020

Published online: 15 April 2020

\section{References}

1. Ferlay J, Soerjomataram I, Dikshit R, et al. Cancer incidence and mortality worldwide: sources, methods and major patterns in GLOBOCAN 2012. Int J Cancer. 2015;136:E359-86.

2. Stewart BW, Wild C. International agency for research on cancer. World cancer report: World Health Organization; 2014.

3. Ruiz i Altaba A. Hedgehog signaling and the Gli code in stem cells, cancer, and metastases. Sci Signal. 2011;4:Pt9.

4. Gulino A, Ferretti E, De Smaele E. Hedgehog signalling in colon cancer and stem cells. EMBO Mol Med. 2009:1:300-2.

5. Stecca B, Mas C, Clement $V$, et al. Melanomas require HEDGEHOG-GLI signaling regulated by interactions between GLI1 and the RAS-MEK/AKT pathways. Proc Natl Acad Sci USA. 2007;104:5895-900.

6. Jones FS, Jones PL. The tenascin family of ECM glycoproteins: structure, function, and regulation during embryonic development and tissue remodeling. Dev Dyn. 2000:218:235-59.

7. Orend G, Chiquet-Ehrismann R. Tenascin-C induced signaling in cancer. Cancer Lett. 2006;244:143-63.

8. Yang Z, Cui Y, Ni W, et al. Gli1, a potential regulator of esophageal cancer stem cell, is identified as an independent adverse prognostic factor in esophageal squamous cell carcinoma. J Cancer Res Clin Oncol. 2017;143:243-54.

9. Yang Z, Zhang C, Qi W, et al. GLI1 promotes cancer stemness through intracellular signaling pathway PI3K/Akt/NFkB in colorectal adenocarcinoma. Exp Cell Res. 2018;373:145-54.

10. Parekh K, Ramachandran S, Cooper J, et al. Tenascin-C, over expressed in lung cancer down regulates effector functions of tumor infiltrating lymphocytes. Lung Cancer. 2005;47:17-29.

11. Yang $Z T$, Yeo $S Y$, Yin $Y X$, et al. Tenascin-C, a prognostic determinant of esophageal Squamous cell carcinoma. PLoS ONE. 2016;11:1-17.

12. Jachetti $E$, Caputo S, Mazzoleni S, et al. Tenascin-C protects cancer stemlike cells from immune surveillance by arresting T-cell activation. Cancer Res. 2015;75:2095-108.

13. Wang J, Lu F, Ren Q, et al. Novel histone demethylase LSD1 inhibitors selectively target cancer cells with pluripotent stem cell properties. Cancer Res. 2011;71:7238-49.

14. Zhang X, Lu F, Wang J, et al. Pluripotent stem cell protein Sox2 confers sensitivity to LSD1 inhibition in cancer cells. Cell Rep. 2013;5:445-57.

15. Amente S, Lania L, Majello B. The histone LSD1 demethylase in stemness and cancer transcription programs. Biochim Biophys Acta. 2013;1829:981-6.

16. Nie S, Gurrea M, Zhu J, et al. Tenascin-C: a novel candidate marker for cancer stem cells in glioblastoma identified by tissue microarrays. J Proteome Res. 2015;14:814-22.

17. Cai J, Du S, Wang H, et al. Tenascin-C induces migration and invasion through JNK/C-Jun signalling in pancreatic cancer. Oncotarget. 2017:8:74406-22

18. Santini R, Vinci MC, Pandolfi S, et al. HEDGEHOG-GLI signaling drives self-renewal and tumorigenicity of human melanoma-initiating cells. Stem Cells. 2012;30:1808-18.

\section{Publisher's Note}

Springer Nature remains neutral with regard to jurisdictional claims in published maps and institutional affiliations. 